Results Results have shown a reduction in missed administration of drugs, a reduction in drug errors, both in prescribing and administration, increased speed of response for discharge prescriptions and a cost reduction in the pharmacy budget to the hospice.

Conclusion Electronic prescribing is a safe and efficient method for the prescribing and administration of drugs within an independent hospice.

\section{P-127 IMPROVING ADMINISTRATION TIME OF CONTROLLED DRUGS}

Jimmy Brash, Alison Bacon, George Coxon, Julie Cuckson, Andrew Fletcher, Tracy Parkinson, Debbie Seed, Barbara Sutton, Jayne Wilkinson. St Catherine's Hospice, Lancashire, UK

\subsection{6/bmispcare-2017-hospice.152}

Background In response to a perceived increase in patient needs and associated increase in workload, a simple analysis of nursing activity highlighted a need to revisit working practices and procedures. Of particular note for this poster was that each day 15 hours of registered nurse (RN) time was dedicated to the administration of 'as required'/PRN controlled drugs (CDs) along with additional (unmeasured) time required to administer those on a recurring prescription.

Aims

- Optimise symptom management

- Administer CDs alongside other medications

- Improve response time to requests for PRN CDs

- Create efficiencies in RN time management.

Methods

- Established a time limited working party to consider and modernise CD working practices. The group comprised:

- Two staff nurses

- Medical representation

- Ward manager and sisters

- CD Accountable Officer/registered manager

- Consider relevant aspects of The Misuse of Drugs Regulations 2001, Controlled drugs regulations and associated guidance

- Developed a robust and safe approach to CD management

- In conjunction with NHS England, a thorough risk assessment was completed.

Results A system has been devised which will make greater use of named patient CDs (oral/transdermal) rather than 'stock' CDs; this will enable:

- Storage of named patient CDs (regular prescriptions and PRN) in high quality medicines cupboards at each individual patient's bedside

- Secure and prompt access to the cupboards by registered nurses

- Single nurse administration of these CDs supported by a robust system of checks and balances

- Fully revised Standard Operating Procedures (CDs)

- Scope does not include parenteral medications.

Conclusion Benefits:

- Rapid access to PRN medications when needed

- Timely administration of regular prescription CDs and nonCDs; thus, a more responsive approach to much needed symptom management

- Reduced time locating stock cupboard keys

- Reduced risk of incorrect administration.

\section{P-128 A QUALITATIVE ANALYSIS OF THE EXPERIENCE OF EPISODIC BREATHLESSNESS IN ADVANCED LUNG CANCER}

${ }^{1}$ Emily Stowe, ${ }^{2}$ Richard Wagland. ${ }^{1}$ St Clare Hospice, Essex, UK; ${ }^{2}$ University of Southampton, Southampton, UK

\subsection{6/bmjspcare-2017-hospice.153}

Background Episodic breathlessness is a common and distressing symptom for people with advanced lung cancer, impacting carers as well as patients. Previous research has quantified the symptom occurrence of episodic breathlessness, identifying the average frequency, duration and severity of episodes, but rarely explored the lived experience of these different aspects. More understanding of the distress associated specifically with episodic breathlessness would inform more appropriate treatment strategies.

Aims To explore the nature of the distress associated with episodic breathlessness and the extent to which each aspect is bothersome for people with advanced lung cancer.

Methods Semi-structured interviews, analysed with an interpretative phenomenological approach, were conducted with four participants recruited from a hospice in Essex. All participants had advanced lung cancer. Ethical approval was secured from NRES Committee London - Hampstead (15/LO/1423) prior to the start of the study.

Results A multifaceted relationship existed between participants' perceptions of duration, frequency and severity in episodic breathlessness. The following key themes emerged: impact on self; searching for answers; impact of previous experience; emotional work of episodic breathlessness; difficulty in quantifying different aspects; identifying most bothersome aspects. Patients benchmarked severity against previous episodes. The unpredictability of severity and duration and constant assessment of potential triggers causing frequency engendered anxiety. Participants appeared to conflate severity and duration more than frequency, but one aspect often impacted a participant's perceptions of the other two.

Conclusions Findings suggest a complex, subjective relationship between different aspects of episodic breathlessness. The importance of considering the whole symptom experience, rather than just symptom occurrence is highlighted. The emotional work of episodic breathlessness should be considered in its management, as well as the interaction between different aspects. Clinicians should be aware of differences between individuals and seek to understand their lived experiences of episodic breathlessness to ensure appropriate treatment of this difficult symptom.

\section{P-129 THE USE OF RELAXATION, GUIDED IMAGERY AND VISUALISATION TECHNIQUES TO HELP RELIEVE ANXIETY AND PAIN IN PALLIATIVE CARE}

Jan Proud. St Margaret's Hospice, Somerset, UK

10.1136/bmjspcare-2017-hospice.154

Non-pharmacological interventions are not always considered as a primary or concurrent treatment for complex physical symptoms. Work carried out within the hospice setting has shown marked improvements in the physical and psychological condition of patients who have difficult to control symptoms, by using alternative patient-centred, holistic techniques. 
The hospice employed an autogenic psychotherapist to treat a patient who was in a chronic state of stress. It was identified that stress management would have a profound effect on the patient's overall condition. Teaching sessions were provided to staff enabling them to use techniques that have shown positive symptom management results.

Conventional practice is to treat with medication - analgesia and anxiolytics. The aim was to introduce a variety of techniques giving both patients and their carers strategies to use to help relieve symptoms of anxiety and pain within palliative care.

Training sessions included experiential learning and reflective practice, taught staff to use simple breathing exercises, progressive muscular relaxation, guided imagery and visualisation techniques.

Staff discovered a powerful tool to help patients and their carers to take control of their own situation, using non pharmacological interventions to give control over physical responses to symptom management.

Using a toolkit of techniques, patients are supported to use the techniques on a face-to-face basis, using technology with CDs, and personalised recordings that they can use when and where required.

Enabling patients when they feel most out of control has shown improvements in psychological distress and physical symptom control. Staff have felt more empowered and able to introduce accessible psychological interventions alongside conventional pharmacological prescriptions.

\section{P-130 A TABOO SYMPTOM? ASSESSING MALODOUR FROM MALIGNANT FUNGATING WOUNDS}

${ }^{1}$ Mandy Stamper, ${ }^{2}$ Laura Green, ${ }^{2}$ Louise Wem. ' Overgate Hospice, Elland, Halifax, West Yorkshire; ${ }^{2}$ University of Bradford, Bradford, UK

\subsection{6/bmispcare-2017-hospice.155}

Background Symptom control of malodour in patients with Malignant Fungating Wounds (MFW) can be challenging as there is little evidence or guidelines. Malodour can be a taboo in society. Human response to smell is complex. A literature review showed that malodour is a major concern to patients, families and staff. As a nurse working in a hospice, I wanted to look at how we could develop a standard for assessing malodour.

Aim My presentation shares my experience in setting a standard for auditing the assessment of the symptom of malodour for patients with MFWs in a UK hospice. I chose this area because malodour from MFWs is a challenge in practice.

Methods I carried out a literature search to see what evidence and guidelines there were to inform my audit standard. I analysed the information and reflected on my 11 years' experience as a hospice nurse.

Results Patients with the symptom of malodour from MFW are affected physically, emotionally and socially. The symptom also affects their family and friends and can be distressing for other patients and staff. I felt the practice of assessing malodour at the hospice could improve so would start a clinical audit with my standard that;

'All patients that are admitted to the hospice with a malignant fungating wound will have the symptom of malodour holistically assessed to support the development of a patientcentred plan of care aimed at improving quality of life.'

Conclusions I was then able to audit the assessment of malodour as a basis for change in our practice aims at improving the management of this distressing symptom. The results have informed my leading a multidisciplinary group to devise a holistic assessment tool for assessing the symptoms of malignant fungating wounds.

\section{P-131 HOSPITAL COMPLIANCE WITH NICE GUIDANCE - INITIATING STRONG OPIOIDS FOR PAIN IN PALLIATIVE CARE}

Deborah Lam, Jennie Pickard, Mona Shalabi, Sophie Harrison, Samantha Kay. University Hospital of South Manchester NHS Foundation Trust, Manchester, UK

10.1136/bmjspcare-2017-hospice. 156

Background When strong opioids are initiated for pain in patients receiving palliative care, practice varies. As a result, there is a risk that patients will receive either sub-therapeutic or potentially toxic doses of opioids. Choice of formulation is important from a patient concordance and cost perspective. In May 2012, the National Institute for Health and Clinical Excellence (NICE) published guidelines on initiating strong opioids for adults with advanced and progressive disease ('Opioids in palliative care: safe and effective prescribing of strong opioids for pain in palliative care of adults'). We reviewed the prescribing practice on hospital wards and assessed our compliance with the guidance and considered the barriers to compliance.

Aim To assess compliance with NICE guidance when initiating strong opioids for pain in adults with a life-limiting illness.

Methods We identified 20 patients with a life limiting illness and that were initiated on strong opioids for pain during their current hospital admission. We reviewed the medical notes and prescriptions measuring prescribing practice against 10 standards outlined by NICE. The patients were from 12 wards including medical and surgical wards. The data was collected over three months.

Results Out of the 10 standards, compliance was 100\% in three standards, $50 \%$ or above in six standards, and less than $50 \%$ in one standard.

Conclusions The results highlighted areas for improvement. The main barrier appeared to be lack of awareness of the guidance. Due to the high turnover of medical staff, continued education is crucial. We have developed an e-learning module, highlighting the guidance, which will be part of the Trust's mandatory training. The Trust is implementing electronic prescribing and endeavours to include alerts when opioids are prescribed to encourage compliance. With improved compliance, prescribing practice will be safer and the management of pain in our patients will be more effective.

\section{P-132 APPROPRIATE AND INAPPROPRIATE USE OF NALOXONE IN PALLIATIVE CARE - TWO CASE REPORTS FROM A HOSPITAL}

Deborah Lam, Jennie Pickard, Sophie Harrison, Samantha Kay. University Hospital of South Manchester NHS Foundation Trust, Manchester

10.1136/bmjspcare-2017-hospice. 157 